ESAIM: PROCEEDINGS AND SURVEYS, 2017, Vol. 57, p. 48-63

Oana Silvia Serea \& Walter Briec Editors

\title{
SOME GENERAL PRINCIPLES IN TROPICAL CONVEXITIES
}

\author{
Charles Horvath ${ }^{1}$
}

\begin{abstract}
We present what the title says with an emphasis on separation and topological properties as they relate to some of the fundamental principles of nonlinear analysis.
\end{abstract}

\section{INTRODUCTION}

The text is structured along the lines of a short talk given at ETAMM 2016. Some details are given in the first part, which is mainly algebraic or set theoretical; few details are given in the second part where algebra and topology intertwine. The first part revolves maily around the Kakutani Separation Property, also known as the Stone-Kakutani Property or the algebraic Hahn-Banach; going into the analytic or the geometric Hahn-Banach would have taken us too far. The second part revolves around a basic intersection theorem, the theorem of Knaster-Kuratowski-Mazurkiewicz - which is an avatar of Brouwer's Fixed Point Theorem - and some of its consequences, mainly fixed point theorems for single valued or multivalued maps. We refrained from giving the most general statement or the most general proof, when we gave one at all. With the last part we come back to the first part of the paper; the result stated their shows that the Kakutani Separation Prop erty, along with some natural topological properties, like connectedness of the convex sets, are at the heart of the fixed points results - and others too - that were presented in the preceding section. We have not tried to be exhaustive duality and variational inequalities in tropical convexities are not to be found here - we tried to show on a few basic results why and how things work.

Applications are made mainly to the standard tropical convexities, maxplus in $\mathbb{R}^{n}$ and maxtimes, also called $\mathbb{B}$-convexity, in $\mathbb{R}_{+}^{n}$. Some infinite dimensional tropical convexities are listed among the examples but they are not studied here. The topological structures of some infinite dimensional spaces naturally related to tropical convexity are studied in [3] and [4].

\section{Convexities And CONVEX SPACES}

\subsection{The general framework}

Definition 2.1.1. A convexity on a set $X$ is a family $\mathcal{C}$ of subsets of $X$ such that:

(Conv1) $\emptyset \in \mathcal{C}$ and $X \in \mathcal{C}$ and, $\forall x \in X,\{x\} \in \mathcal{C}$;

(Conv2) if $\mathcal{A}$ is a subfamily of $\mathcal{C}$ then $\bigcap \mathcal{A}$ belongs to $\mathcal{C}$;

(Conv3) if $\mathcal{A}$ is an updirected ${ }^{1}$ subfamily of $\mathcal{C}$ then $\bigcup \mathcal{A}$ belongs to $\mathcal{C}$.

Elements of $\mathcal{C}$ are called convex sets and $(X, \mathcal{C})$ is a convex space.

${ }^{1}$ Université de Perpignan, Département de Mathématiques, Laboratoire LAMPS, horvath@univ-perp.fr

${ }^{1} \forall C, C^{\prime} \in \mathcal{C} \quad \exists C^{\prime \prime} \in \mathcal{C}$ such that $C \cup C^{\prime} \subset C^{\prime \prime}$.

(C) EDP Sciences, SMAI 2017 
Given $A \subset X$ let $[A]_{\mathcal{C}}=\bigcap\{C \in \mathcal{C}: A \subset C\} ;$ It is the $\mathcal{C}$-hull of $A$.

By Conv1 and Conv2, $\forall A \subset X \quad[A]_{\mathcal{C}} \in \mathcal{C}$ and $\left[A \rrbracket_{\mathcal{C}}=A \Leftrightarrow A \in \mathcal{C}\right.$. Furthermore,

$$
\forall A, B \subset X \quad \begin{cases}(1) & A \subset[A]_{\mathcal{C}} \\ (2) & A \subset B \Rightarrow[A]_{\mathcal{C}} \subset[B]_{\mathcal{C}} \\ (3) & \left.[\llbracket A]_{\mathcal{C}}\right]_{\mathcal{C}}=[A]_{\mathcal{C}}\end{cases}
$$

For all non empty sets $S$ let $\langle S\rangle$ be the family of nonempty finite subsets of $S$. Condition (Conv3) implies that

Lemma 2.1.2. For all convex space $(X, \mathcal{C})$ and for all subsets $A$ of $X$

$$
\left[A \rrbracket_{\mathcal{C}}=\bigcup_{F \in\langle A\rangle}[F]_{\mathcal{C}}\right.
$$

Proof. We can assume that $A \neq \emptyset$. The family $\langle A\rangle$ of nonempty finite subsets of $A$ is updirected and consequently so is the family of convex sets $\left.\{\llbracket F]_{\mathcal{C}}: F \in\langle A\rangle\right\}$ and therefore $\bigcup_{F \in\langle A\rangle}\left[F \rrbracket_{\mathcal{C}}\right.$ is convex, call this set $C$. If $F \in\langle A\rangle$ then $F \subset[F]_{\mathcal{C}} \subset[A]_{\mathcal{C}}$ from which $C \subset[A]_{\mathcal{C}}$. From $A=\bigcup_{F \in\langle A\rangle} F$ we have $A \subset C$ and consequently, since $C \in \mathcal{C}, \llbracket A]_{\mathcal{C}} \subset C$. We have shown that $[A]_{\mathcal{C}}=C$.

Lemma 2.1.3. For all convex space $(X, \mathcal{C})$ and for all subset $C$ of $X, C \in \mathcal{C}$ if and only if, for all finite subset $F$ of $C,[F]_{\mathcal{C}} \subset C$.

Proof. This is a restatement of Lemma 2.

The $\mathcal{C}$-hull of a finite set is a $\mathcal{C}$-polytope. From 1 and 2 we have

$$
\forall C \in \mathcal{C} \quad C=\bigcup_{\substack{P \subset C \\ P \text { is a polytope }}} P
$$

Conditions 1 say that the application $A \mapsto[A]_{\mathcal{C}}$ is a Moore closure operator while 2 says that it is an algebraic closure operator. One can easily check, that given a algebraic Moore closure operator $[\cdot]: \mathcal{P}(X) \rightarrow \mathcal{P}(X)$ on a set $X$ such that $\llbracket \emptyset]=\emptyset$ and, forall $x \in X,[\{x\}]=\{x\}$, the set of fixed points of [ $\cdot]$ is a convexity $\mathcal{C}$ on $X$ for such that $\llbracket \cdot]_{\mathcal{C}}=[\cdot]$.

From here on, if no confusion is possible, the convex hull operator associated to a given convexity will mostly be written [ $]$ and we will write $\left[p_{1}, \cdots, p_{m} \rrbracket\right.$ for $\left[\left\{p_{1}, \cdots, p_{m}\right\}\right]$.

Definition 2.1.4. A convex space $(X, \mathcal{C})$ is recursive if, for all non empty finite subset $S$ of $X$ and for all point $p_{0} \in X$

$$
\left[S \cup\left\{p_{0}\right\} \rrbracket=\bigcup_{p \in[S]}\left[p, p_{0}\right] .\right.
$$

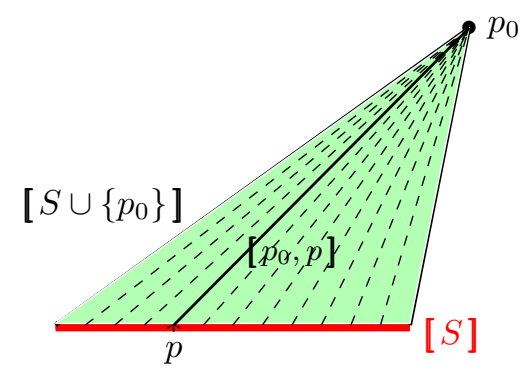


Let us say that a convexity $\mathcal{C}$ on a set $X$ is an interval convexity if, for all $C \subset X$, the following two conditions are equivalent :

$$
\begin{cases}(1) & C \in \mathcal{C} \\ (2) & \forall p, q \in C \quad[p, q] \subset C\end{cases}
$$

Lemma 2.1.5. A recursive convexity $\mathcal{C}$ on a set $X$ is an interval convexity.

Proof. Let $S=\left\{p_{0}, \cdots, p_{m}\right\}$ be an arbitrary non empty subset of $C$. Assume that, for all $p, q \in C, \llbracket p, q \rrbracket \subset C$. We show, by induction on the cardinality of $S$, that $[S] \subset C$. If $m \leq 1$ there is nothing to show. Assume that $m>1$; from $D=\left[S \backslash\left\{p_{m}\right\}\right] \subset C$ and from $[S]=\cup_{q \in D}\left[p_{m}, q\right]$ we have $\left.\llbracket S\right] \subset C$; by Lemma 2.1.3, $C$ is convex;

Definition 2.1.6. A convex space $(X, \mathcal{C})$ has Property $\mathbf{C}$ if, for all triple $\left(a, b_{1}, b_{2}\right)$ of points of $X$ and all points $c \in \llbracket a, b_{1} \rrbracket$ and $d \in \llbracket c, b_{2} \rrbracket$ there exists a point $b \in\left[b_{1}, b_{2} \rrbracket\right.$ such that $d \in[a, b]$

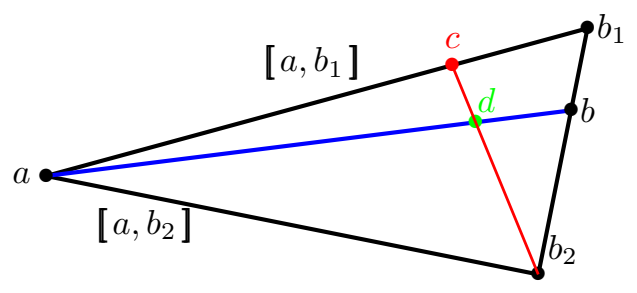

Proposition 2.1.7. A convexity $\mathcal{C}$ on a set $X$ is recursive if and only if it is an interval convexity with Property $C$.

Proof. Assume that $\mathcal{C}$ is recursive, by Lemma 2.1.5 it is an interval convexity. Let the points $a, b_{1}, b_{2}, c$ and $d$ be as in definition 2.1.6. From $\left[a, b_{1}, b_{2}\right]=\bigcup_{b \in\left[b_{1}, b_{2}\right]}[a, b]$ and $d \in \llbracket c, b_{2} \rrbracket \subset\left[a, b_{1}, b_{2}\right]$ there exists $b \in\left[b_{1}, b_{2}\right]$ such that $d \in[a, b]$.

Assume now that $\mathcal{C}$ is an interval convexity for which Property $\mathrm{C}$ holds and let us show that $\mathcal{C}$ is recursive. Since $S_{0} \cup\left\{p_{0}\right\} \subset \bigcup_{p \in\left[S_{0}\right]} \llbracket p, p_{0} \rrbracket$ it is enough to see that $\left.\bigcup_{p \in\left[S_{0}\right]} \llbracket p, p_{0}\right]$ is convex since we then have $\llbracket S_{0} \cup\left\{p_{0}\right\} \rrbracket \subset$ [ $\left.\bigcup_{p \in\left[S_{0}\right]}\left[p, p_{0}\right]\right]=\bigcup_{p \in\left[S_{0}\right]}\left[p, p_{0}\right]$.

Let $p_{1}, p_{2}$ be two points of $\bigcup_{p \in\left[S_{0}\right]}\left[p, p_{0}\right]$. We show that $\left[p_{1}, p_{2}\right] \subset \bigcup_{p \in\left[S_{0}\right]}\left[p, p_{0}\right]$.

There exist $q_{1}, q_{2} \in\left[S_{0}\right]$ such that $p_{i} \in\left[p_{0}, q_{i}\right]$; let $c \in\left[p_{1}, p_{2}\right]$.

Applying Property $\mathrm{C}$ to the configuration $\left\{\begin{array}{l}a=p_{0} \\ c_{1}=p_{1} \\ b_{1}=q_{1} \\ c_{2}=b_{2}=p_{2}\end{array}\right.$

we find a point $b^{\prime} \in\left[q_{1}, p_{2}\right]$ such that $c \in\left[p_{0}, b^{\prime}\right]$.

With the configuration $\left\{\begin{array}{l}a=p_{0} \\ c_{1}=b_{1}=q_{1} \\ b_{2}=q_{2} \\ c_{2}=p_{2}\end{array} \quad\right.$ we find a point $b \in\left[q_{1}, q_{2}\right]$ such that $b^{\prime} \in\left[p_{0}, b\right]$.

From $c \in\left[p_{0}, b^{\prime}\right]$ and $b^{\prime} \in\left[p_{0}, b\right]$ we have $c \in\left[p_{0}, b\right]$.

From $\left.b \in \llbracket q_{1}, q_{2}\right]$ and $q_{1}, q_{2} \in\left[S_{0}\right]$ we have $\left.b \in \llbracket S_{0}\right]$ and finally $c \in \bigcup_{p \in\left[S_{0}\right]}\left[p, p_{0}\right]$ and therefore $\left[p_{1}, p_{2} \rrbracket \subset\right.$ $\bigcup_{p \in\left[S_{0}\right]}\left[p, p_{0}\right]$. 
Given a set $X$ let $X^{\{0,1\}}$ be the family of non empty finite subsets of $X$ of cardinality at most 2 ; given a $\operatorname{map} \mathcal{I}: X^{\{0,1\}} \rightarrow \mathcal{P}(X) \backslash\{\emptyset\}$ let

$$
\mathcal{C}_{\mathcal{I}}=\{C \subset X: \forall\{x, y\} \subset C \quad \mathcal{I}(\{x, y\}) \subset C\}
$$

The proofs of Lemmas 2.1.8 and 2.1.9 below are left to the reader.

Lemma 2.1.8. $\left(X, \mathcal{C}_{\mathcal{I}}\right)$ is a convex space if and only if the following condition holds:

$\mathbf{L}_{\mathbf{0}}: \forall x \in X \mathcal{I}(\{x\})=\{x\}$

in which case it is an interval convexity.

The convex interval $\llbracket x, y]_{\mathcal{C}_{\mathcal{I}}}$, which is the intersection of all the $\mathcal{C}_{\mathcal{I}}$ convex sets containing $\{x, y\}$, does not have to be $\mathcal{I}(\{x, y\})$.

Lemma 2.1.9. Assume that condition $\mathbf{L}_{0}$ holds. The following statements are equivalent:

(1) For all $\{x, y\} \subset X\left[x, y \rrbracket_{\mathcal{C}_{\mathcal{I}}}=\mathcal{I}(\{x, y\})\right.$.

(2) For all $\{x, y\} \subset X$ and for all $\{w, z\} \subset \mathcal{I}(\{x, y\}), \mathcal{I}(\{w, z\}) \subset \mathcal{I}(\{x, y\})$.

General convex spaces are more akin to general topological spaces or measure spaces, in that the structure is defined by a family of subsets, than to the usual linear convex sets where the convex structure is associated to some previously defined algebraic structure. This of course does not preclude a given convexity structure from being associated to an underlying algebraic structure as, for example, it is the case for tropical, or linear, convexity. In any case, in the absence of an underlying primary structure from which the convexity structure is derived, it seems natural to define a morphism of convex spaces as it is done for topological spaces (continuous maps) or measure spaces (measurable maps) ; morphisms of general convex spaces are called, following Van Mill and Van de Vell, convexity preserving maps. A convexity preserving map from a convex space $\left(X_{1}, \mathcal{C}_{1}\right)$ to a convex space $\left(X_{2}, \mathcal{C}_{2}\right)$ is a map $\varphi: X_{1} \rightarrow X_{2}$ such that, for all $C \in \mathcal{C}_{2}, \varphi^{-1}(C) \in \mathcal{C}_{1}$.

Obviously, the class of convex spaces and convexity preserving maps constitute a category, whose study is not the subject matter of this paper. All we will need is the associated notion of isomorphism : two convex spaces $\left(X_{1}, \mathcal{C}_{1}\right)$ and $\left(X_{2}, \mathcal{C}_{2}\right)$ are isomorphic if there exists a bijective convexity preserving map $\varphi: X_{1} \rightarrow X_{2}$ whose inverse is also convexity preserving; such a map is naturally called an isomorphism of convexity spaces. Clearly, if $\varphi:\left(X_{1}, \mathcal{C}_{1}\right) \rightarrow\left(X_{2}, \mathcal{C}_{2}\right)$ is an isomorphism then so is $\varphi^{-1}:\left(X_{2}, \mathcal{C}_{2}\right) \rightarrow\left(X_{1}, \mathcal{C}_{1}\right)$ and $\varphi:\left(X_{1}, \mathcal{C}_{1}\right) \rightarrow\left(X_{2}, \mathcal{C}_{2}\right)$ is an isomorphism if and only if it is bijective and for all $C_{1} \in \mathcal{C}_{1}$ all $C_{2} \in \mathcal{C}_{2}, \varphi\left(C_{1}\right) \in \mathcal{C}_{2}$ and $\varphi^{-1}\left(C_{2}\right) \in \mathcal{C}_{1}$.

\subsection{Algebraic separation of convex sets}

Definition 2.2.1. A convex space $(X, \mathcal{C})$ has the Pash-Peano Property if, for all quintuple $\left(a, b_{1}, b_{2}, c_{1}, c_{2}\right)$ of points of $X$ such that $c_{i} \in \llbracket a, b_{i} \rrbracket$, one has

$$
\left[b_{1}, c_{2}\right] \cap\left[b_{2}, c_{1}\right] \neq \emptyset .
$$

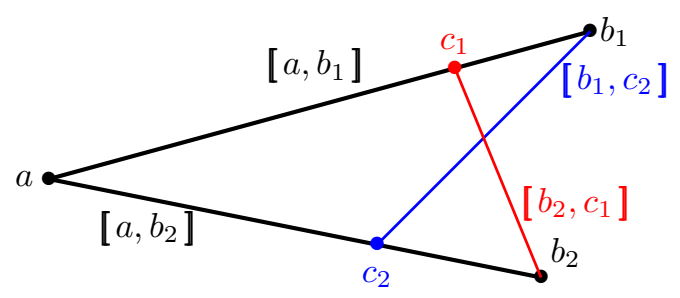

Definition 2.2.2. A half-space of a convex space $(X, \mathcal{C})$ is a convex set $H \in \mathcal{C}$ whose complement $X \backslash H$ is also in $\mathcal{C}$. 
Definition 2.2.3. A convex set $(X, \mathcal{C})$ has the Kakutani Property if, for all pair $\left(C_{1}, C_{2}\right)$ of disjoint non empty convex sets there exists a half-space $H$ such that $C_{1} \subset H$ and $C_{2} \subset X \backslash H$.

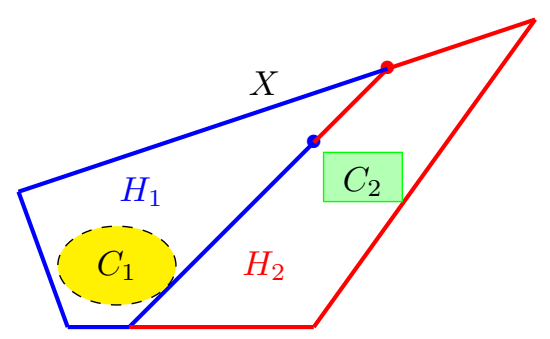

Kakutani Property

Theorem 2.2.4. A recursive convex space $(X, \mathcal{C})$ has the Kakutani Property if and only if it has the Pash-Peano Property.

Proof. A proof can be found in [20].

Corollary 2.2.5. If an interval convexity $\mathcal{C}$ on a set $X$ has Property $C$ and the Pash-Peano Property then it has the Kakutani Property.

Proof. By Proposition 2.1.7.

Both Property C and the Pash-Peano Property are properties of polytopes spanned by at most three points; one could informally say that, for interval convexities, the Kakutani property is a "2-dimentional property". One can compare with Proposition 2.2.8 below.

Definition 2.2.6. A convex space $(X, \mathcal{C})$ has the Generalized Pash-Peano

Property if for all non empty finite subsets $B_{1}$ and $B_{2}$ of $X$ and all triple of points $\left(a, c_{1}, c_{2}\right)$ with $\left.c_{i} \in \llbracket\{a\} \cup B_{i}\right]$ one has $\left[\left\{c_{1}\right\} \cup B_{2}\right] \cap\left[\left\{c_{2}\right\} \cup B_{1}\right] \neq \emptyset$.

A proof of the following proposition can be found in [20].

Proposition 2.2.7. A convex space $(X, \mathcal{C})$ has the Kakutani Property if and only if it has the Generalized Pash-Peano Property.

Given a convex space $(X, \mathcal{C})$ and a convex set $P \in \mathcal{C}$ the family $\{P \cap C: C \in \mathcal{C}\}$ is a convexity on $P$ called the induced convexity. Proposition 2.2.8 below is due to Keimel and Wieczorek; their proof can be found [23].

Proposition 2.2.8. A convex space $(X, \mathcal{C})$ has the Kakutani Property if and only if all polytopes have, with respect to the induced convexity, the Kakutani Property.

Proof. Notice that the Generalized Pash-Peano Property holds if and only if it holds on polytopes.

Property C is due to Coppel, [14] where some of the results stated here, albeit in a somewhat different form and for a different purpose can also be found. Convexities with the Kakutani Property are studied in [14] and [29]; Holmes in [17] explicitely uses the Pash-Peano Property - without giving it a name since in a real vector space it is obvious - to prove the algebraic separation theorem. 


\section{TROPICAL CONVEXITY}

A semiring is a set $A$, with two operations $\oplus: A \times A \rightarrow A$ and $\odot: A \times A \rightarrow A$ and two distinguished elements $1_{A}$ and $0_{A}$ such that:

(1) $\left(A, \oplus, 0_{A}\right)$ is an abelian semigroup.

(2) for all $x, y, z \in A, z \odot(x \odot y)=(z \odot x) \odot y$.

(3) for all $x, y, z \in A, z \odot(x \oplus y)=(z \odot x) \oplus(z \odot y)$.

(4) for all $x \in A, 0_{A} \odot x=0_{A}$ and $1_{A} \odot x=x$

For simplicity we will assume that $\odot$ is commutative.

An idempotent semiring, also called a tropical semiring or a Maslov semiring is a semiring $A$ such that, for all $x \in A, x \oplus x=x$.

A (tropical) semifield is a (tropical) semiring for which $\left(A_{\star}, \odot, 1_{A}\right)$ is a group $\left(A_{\star}=A \backslash\left\{0_{A}\right\}\right)$. Since tropical multiplication can sometimes be the "usual addition" or the "usual multiplication" of scalars we will denote, for an arbitrary semifield $\left(A_{\star}, \odot, 1_{A}\right)$, by $u^{\text {inv }}$ the inverse of $u \in A_{\star}$.

An idempotent abelian semigroup $\left(A, \oplus, 0_{A}\right)$ is a semilattice with smallest element $0_{A}$, and reciprocally, where $x \oplus y$ is the least upper bound of $\{x, y\}$ with respect to the partial order $x \leqslant y$ if $x \oplus y=y$.

Some tropical semirings

(1)(maxplus semiring) $A=\mathbb{R} \cup\{-\infty\}, 0_{\max +}=-\infty, 1_{\max +}=0, x \oplus y=\max \{x, y\}, x \odot y=x+y$.

On could could also take $A$ to be $\mathbb{Z} \cup\{-\infty\}$ or $\mathbb{Q} \cup\{-\infty\}$.

(2)(maxtimes semiring) $A=\mathbb{R}_{+}, 0_{\max \times}=0,1_{\max \times}=1, x \oplus y=\max \{x, y\}, x \odot y=x y$. One could also take for $A \mathbb{N}$ or $\mathbb{Q}_{+}$.

(3) (distributive lattice) $\left(L, \vee, \wedge, 0_{L}, 1_{L}\right)$ is a disributive lattice with a smallest and a largest element; let $x \oplus y=$ $x \vee y$ and $x \odot y=x \wedge y$.

The maxplus and the maxtimes semirings are semifields; if $u \in \mathbb{R}$ the multiplicative inverse of $u$ in the maxplus semifield is $-u$; if $u \in \mathbb{R}_{+} \backslash\{0\}=\mathbb{R}_{++}$the multiplicative inverse of $u$ in the maxtimes structure is $u^{-1}$.

A (tropical) semimodule over a (tropical) semiring $\left(A, \oplus_{A}, \odot_{A}, 0_{A}, 1_{A}\right)$ is an abelian (idempotent) semigroup $\left(M, \oplus, 0_{M}\right)$ endowed with an operation $\odot: A \times M \rightarrow M$ such that :

$$
\forall a, b \in A \quad \forall x, y \in M\left\{\begin{array}{l}
a \odot(x \oplus y)=(a \odot x) \oplus(b \odot y) \\
\left(a \oplus_{A} b\right) \odot x=(a \odot x) \oplus(b \odot x) \\
(a \odot A) \odot x=a \odot(b \odot x) \\
1_{A} \odot x=x \text { and } 0_{A} \odot x=0_{M}
\end{array}\right.
$$

$>$ From here on we will mostly drop the subscripts on the operations $\oplus, \odot$. Tropical semirings are also called idempotent semirings or Maslov semirings.

A tropical semiring $A$ is a totally ordered tropical semiring if for all $x, y \in A, x \oplus y \in\{x, y\}$.

The maxplus semiring $\mathbb{A} \cup\{-\infty\}$ and the maxtimes times semiring $\mathbb{A}_{+}$where $\mathbb{A} \in\{\mathbb{Z}, \mathbb{Q}, \mathbb{R}, \cdots\}$ are totally ordered.

\section{Some tropical semimodules}

(1) Let $A$ be an arbitrary semiring and $S$ a non empty set. On $M=A^{S}$, the set of all functions from $S$ to $A$, let $\oplus$ and $\odot$ be the pointwise operations: $(f \oplus g)(s)=f(s) \oplus g(s)$ and $(a \odot f)(s)=a \odot f(s)$; and take $0_{M}$ be the function which is identically $0_{A}$. 
(1.1) If $A$ is the maxplus semiring $\mathbb{R} \cup\{-\infty\}$ and if $S=\{1, \cdots, n\}$ we have the maxplus semimodule ( $\mathbb{R} \cup$ $\{-\infty\})^{n}$;

(1.2) If $A$ is the maxtimes semiring $\mathbb{R}_{+}$and $S$ is as above we have the maxtimes semimodule $\left(\mathbb{R}_{+}\right)^{n}$.

(2) Let $(L, \vee)$ be a real vector space semilattice, for example the space of real valued bounded functions on a given set $X$ or the set of real continuous functions on a compact set $X$ where $\vee=\max$; on $L_{+}=\{x \in L: 0 \leqslant x\}$ let $x \odot y=x \vee y$ and, for $t \in \mathbb{R}_{+}, t \odot x=t x$. With these operations $L_{+}$is a semimodule over the maxtimes semiring $\mathbb{R}_{+}$.

A subset $C$ of a tropical semimodule $M$ over a tropical semiring $A$ is tropically convex if :

$$
\forall(x, y) \in C^{2} \quad \forall(a, b) \in A^{2} \text { such that } a \oplus b=1 \quad(a \odot x) \oplus(b \odot y) \in C
$$

Proposition 3.0.1. The set of convex sets of a given semimodule $M$ over an arbitrary tropical semiring $A$ is an interval convexity on $M$ for which, for all $x, y \in M,[x, y]=\left\{(a \odot x) \oplus(b \odot y):(a, b) \in A^{2}\right.$ and $\left.a \oplus b=1\right\}$. Furthermore, for an arbitrary finite and non empty subset $S=\left\{x_{1}, \cdots, x_{m}\right\}$ of $M,[S]=\left\{\bigoplus_{i=1}^{m} a_{i} \odot x_{i}\right.$ : $\left(a_{1}, \cdots, a_{m}\right) \in A^{n}$ s.t. $\left.\bigoplus_{i=1}^{m} a_{i}=1\right\}$

Proof. Let $\mathcal{I}(\{x, y\})=\left\{(a \odot x) \oplus(b \odot y):(a, b) \in A^{2}\right.$ and $\left.a \oplus b=1\right\}$. $\mathbf{L}_{\mathbf{0}}$ holds since, with $a \oplus b=1$, we have $(a \odot x) \oplus(b \odot x)=(a \oplus b) \odot x=1 \odot x=x$.

The following straightforward calculation shows that (2) of Lemma 2.1.9 holds.

Assume that $a_{i} \oplus b_{i}=1$ and $a \oplus b=1$.

Let $w=\left(a_{1} \odot x\right) \oplus\left(b_{1} \odot y\right)$ and $z=\left(a_{2} \odot x\right) \oplus\left(b_{2} \odot y\right)$ with $a_{i} \oplus b_{i}=1$. Then $(a \odot w) \oplus(b \odot z)=$ $\left\{\left[\left(a \odot a_{1}\right) \oplus\left(b \odot a_{2}\right)\right] \odot x\right\} \oplus\left\{\left[\left(a \odot b_{1}\right) \oplus\left(b \odot b_{2}\right)\right] \odot y\right\}$.

$>$ From $\left\{\left[\left(a \odot a_{1}\right) \oplus\left(b \odot a_{2}\right)\right]\right\} \oplus\left\{\left[\left(a \odot b_{1}\right) \oplus\left(b \odot b_{2}\right)\right]\right\}=a \odot\left(a_{1} \oplus b_{1}\right) \oplus b \odot\left(a_{2} \oplus b_{2}\right)=a \odot 1 \oplus b \odot 1=a \oplus b=1$ we have $(a \odot w) \oplus(b \odot z) \in \mathcal{I}(\{x, y\})$.

A similar straightforward calculation yields the second part.

Let $\mathcal{C}_{\text {trop }}(M)$ denote the family of tropically convex subsets of a tropical semimodule $M$ over a tropical semiring $A$; the emptyset belongs to $\mathcal{C}_{\text {trop }}(M)$.

Proposition 3.0.2. If $M$ is a tropical semimodule over a tropical semifield $A$ then tropical convexity on $M$ is recursive.

Proof. . We have seen that $\mathcal{C}_{\text {trop }}(M)$ is an interval convexity; let us see that Property C holds. Let $c$ and $d$ be two points of $M$ such that

which gives

$$
\left\{\begin{array}{l}
(1) \quad c=(s \odot a) \oplus\left(t \odot b_{1}\right) \text { with } s \oplus t=1 \\
\text { and } \\
(2) \quad d=\left(s^{\prime} \odot c\right) \oplus\left(t^{\prime} \odot b_{2}\right) \text { with } s^{\prime} \oplus t^{\prime}=1
\end{array}\right.
$$

$$
d=\left(s^{\prime} \odot s\right) \odot a \oplus\left(\left(s^{\prime} \odot t\right) \odot b_{1}\right) \oplus\left(t^{\prime} \odot b_{2}\right)
$$

If $\left(s^{\prime} \odot t\right) \oplus t^{\prime} \neq 0$ let $u=\left(\left(s^{\prime} \odot t\right) \oplus t^{\prime}\right)^{\text {inv }}$.

Then $\left[\left(s^{\prime} \odot t\right) \odot b_{1} \oplus\left(t^{\prime} \odot b_{2}\right)\right]=\left(\left(s^{\prime} \odot t\right) \oplus t^{\prime}\right) \odot\left(\left(t_{1} \odot b_{1}\right) \oplus\left(t_{2} \odot b_{2}\right)\right)$ with $t_{1}=u \odot\left(s^{\prime} \odot t\right)$ and $t_{2}=u \odot t^{\prime}$. Furthermore, $t_{1} \oplus t_{2}=\left[u \odot\left(s^{\prime} \odot t\right)\right] \oplus\left(u \odot t^{\prime}\right)=u \odot\left[\left(s^{\prime} \odot t\right) \oplus t^{\prime}\right]=1$.

We have shown that $\left.\left(t_{1} \odot b_{1}\right) \oplus\left(t_{2} \odot b_{2}\right) \in \llbracket b_{1}, b_{2}\right]$. Let $b=\left(t_{1} \odot b_{1}\right) \oplus\left(t_{2} \odot b_{2}\right)$.

From $d=\left(s^{\prime} \odot s\right) \odot a \oplus\left(\left(s^{\prime} \odot t\right) \oplus t^{\prime}\right) \odot b$ and $\left(s^{\prime} \odot s\right) \oplus\left(s^{\prime} \odot t\right) \oplus t^{\prime}=s^{\prime} \odot(s \oplus t) \oplus t^{\prime}=s^{\prime} \oplus t^{\prime}=1$ we have $d \in[a, b]$. 
If $\left(s^{\prime} \odot t\right) \oplus t^{\prime}=0$ then $\left(s^{\prime} \odot t\right)=t^{\prime}=0$. Since $A$ is a semifield, either $s^{\prime}=0$ or $t=0$; from $s^{\prime} \oplus t^{\prime}=1$ and $t^{\prime}=0$ we have $s^{\prime}=1$ and therefore $t=0$ and, from $s \oplus t=1, s=1$. So $a=c=d$ and $d=a \oplus(0 \odot b)$ with $b=b_{1}$.

Proposition 3.0.3. If $M$ is a tropical semimodule over a totally ordered tropical semifield $A$ then tropical convexity on $M$ has the Pash-Peano Property.

Proof. Let $c_{i}=\left(s_{i} \odot a\right) \oplus\left(t_{i} \odot b_{i}\right)$ with $s_{i} \oplus t_{i}=1$. We have to show that there exists $\left(u_{1}, v_{1}\right)$ and $\left(u_{2}, v_{2}\right)$ in $A \times A$ such that $\left\{\begin{array}{ll}(1) & u_{1} \oplus v_{1}=u_{2} \oplus v_{2}=1 \\ (2) & \left(u_{1} \odot c_{1}\right) \oplus\left(v_{1} \odot b_{2}\right)=\left(u_{2} \odot c_{2}\right) \oplus\left(v_{2} \odot b_{1}\right)\end{array}\right.$ Substituting for $c_{1}$ and $c_{2}$, respectively, in (2) yields

$\left\{\begin{array}{l}\left(u_{1} \odot c_{1}\right) \oplus\left(v_{1} \odot b_{2}\right)=\left[\left(u_{1} \odot s_{1}\right) \odot a\right] \oplus\left[\left(u_{1} \odot t_{1}\right) \odot b_{1}\right] \oplus\left[v_{1} \odot b_{2}\right] \\ \left(u_{2} \odot c_{2}\right) \oplus\left(v_{2} \odot b_{1}\right)=\left[\left(u_{2} \odot s_{2}\right) \odot a\right] \oplus\left[\left(u_{2} \odot t_{2}\right) \odot b_{2}\right] \oplus\left(v_{2} \odot b_{1}\right)\end{array}\right.$

To complete the proof it is sufficient to find $\left(u_{i}, v_{i}\right)$ such that $\left\{\begin{array}{l}\text { (a) } u_{1} \odot s_{1}=u_{2} \odot s_{2} \\ \text { (b) } u_{1} \odot t_{1}=v_{2} \\ \text { (c) } u_{2} \odot t_{2}=v_{1}\end{array}\right.$ and $u_{1} \oplus v_{1}=u_{2} \oplus v_{2}=1$.

Assume that either $s_{1} \neq 0$ or $s_{2} \neq 0$; let us say $s_{2} \neq 0$.

$$
\text { Given an arbitrary } u_{1} \in A \backslash\{0\} \text { let }\left\{\begin{array}{l}
u_{2}=u_{1} \odot s_{1} \odot s_{2}^{\text {inv }} \\
v_{1}=u_{2} \odot t_{2} \\
v_{2}=u_{1} \odot t_{1}
\end{array}\right.
$$

Equations (a), (b) and (c) hold. We have to see that $u_{1}$ can be chosen such that $u_{1} \oplus v_{1}=u_{2} \oplus v_{2}=1$.

$$
\left\{\begin{array}{l}
u_{1} \oplus v_{1}=u_{1} \oplus\left(u_{2} \odot t_{2}\right)=u_{1} \oplus\left(u_{1} \odot s_{1} \odot s_{2}^{\text {inv }} \odot t_{2}\right)=u_{1} \odot\left[1 \oplus\left(s_{1} \odot s_{2}^{\text {inv }} \odot t_{2}\right)\right] \\
\left.u_{2} \oplus v_{2}=\left[u_{1} \odot s_{1} \odot s_{2}^{\text {inv }}\right] \oplus\left[u_{1} \odot t_{1}\right]=u_{1} \oplus\left[\left(s_{1} \odot s_{2}^{\text {inv }}\right) \oplus t_{1}\right)\right]
\end{array}\right.
$$

Since $A$ is totally ordered and $s_{i} \oplus t_{i}=1$ there are only four cases to consider: $\begin{cases}(1) & s_{1}=s_{2}=1 \\ (2) & s_{1}=t_{2}=1 \\ (3) & t_{1}=s_{2}=1 \\ (4) & t_{1}=t_{2}=1\end{cases}$

Take $u_{1}=1$.

$$
\text { (1) }\left\{\begin{array}{l}
u_{1} \oplus v_{1}=u_{1} \odot\left(1 \oplus t_{2}\right)=u_{1} \odot 1 \\
u_{2} \oplus v_{2}=u_{1} \oplus t_{1}
\end{array}\right.
$$

Take $u_{1}=1$.

$$
\text { (2) }\left\{\begin{array}{l}
u_{1} \oplus v_{1}=u_{1} \odot\left(1 \oplus s_{2}^{\text {inv }}\right) \\
u_{2} \oplus v_{2}=u_{1} \oplus\left(s_{2}^{\text {inv }} \oplus t_{1}\right)
\end{array}\right.
$$

Take $u_{1}=s_{2}$.

$$
\text { (3) }\left\{\begin{array}{l}
u_{1} \oplus v_{1}=u_{1} \odot\left[1 \oplus\left(s_{1} \odot t_{2}\right)\right]=u_{1} \odot 1=u_{1} \\
u_{2} \oplus v_{2}=u_{1}
\end{array}\right.
$$

$$
\left\{\begin{array}{l}
u_{1} \oplus v_{1}=u_{1} \odot\left[1 \oplus\left(s_{1} \odot s_{2}^{\text {inv }}\right)\right] \\
u_{2} \oplus v_{2}=u_{1} \oplus\left(s_{1} \odot s_{2}^{\text {inv }}\right)
\end{array}\right.
$$

If $s_{1} \odot s_{2}^{\text {inv }} \leq 1$ take $u_{1}=1$ and if $1 \leq s_{1} \odot s_{2}^{\text {inv }}$ take $u_{1}=\left(s_{2} \odot s_{1}^{\text {inv }}\right)^{\text {inv }}$. 
Proposition 3.0.4 and Corollary 3.0.5 below follow from the previous propositions of this section and propositions 2.2 .4 and 2.2 .7

Proposition 3.0.4. If $M$ is a tropical semimodule over a totally ordered tropical semifield A then tropical convexity on $M$ has the Kakutani Property and the Generalized Pash-Peano Property.

Corollary 3.0.5. An arbitrary convex subset of a tropical semimodule over a totally ordered tropical semifield is the intersection of all the half spaces containing it.

The structure of halfspaces and separation theorems, algebraic and analytic, in maxplus convexity are studied in [8], [9], [12], [12] and [26].

As is well known, maxplus convexity in $(\mathbb{R} \cup\{-\infty\})^{n}$ and $\mathbb{B}$-convexity (that is maxtimes convexity in $\mathbb{R}_{+}^{n}$ ) are isomorphic: $\ln \left(x_{1}, \cdots, x_{n}\right)=\left(\ln \left(x_{1}\right), \cdots, \ln \left(x_{n}\right)\right)$, with $\ln (0)=-\infty$, is a convexity preserving bijection from $\mathbb{R}_{+}^{n}$ to $(\mathbb{R} \cup\{-\infty\})^{n}$ whose inverse is $\boldsymbol{E}\left(x_{1}, \cdots, x_{n}\right)=\left(e^{x_{1}}, \cdots, e^{x_{n}}\right)$, with $0=e^{-\infty}$, is also convexity preserving.

Since $\mathbb{R}^{n}$ and $\mathbb{R}_{++}^{n}$ are, respectively, convex subsets of the maxplus semimodule $(\mathbb{R} \cup\{-\infty\})^{n}$ and the maxtimes

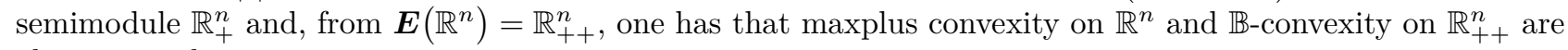
also isomorphic.

\section{More tropical semimodules and tropical convex sets}

Apart from the standard finite dimensional maxplus convexity on $(\mathbb{R} \cup\{-\infty\})^{n}$ or maxtimes convexity $\mathbb{R}_{+}^{n}$ there are natural (possibly) infinite dimensional tropical convexities, for example:

(1) The "Hom functor" yields tropical semimodule: given a two tropical semimodules $M_{1}$ and $M_{2}$ over a tropical semiring $A$ one can consider the set $\operatorname{Hom}_{A}\left(M_{1}, M_{2}\right)$ of all maps $\psi: M_{1} \rightarrow M_{2}$ such that

$\begin{cases}(1) \forall(a, x) \in A \times M_{1} & \psi(a \odot x)=a \odot \psi(x) \\ (2) \forall\left(x_{1}, x_{2}\right) \in M_{1} \times M_{1} & \psi\left(x_{1} \oplus x_{2}\right)=\psi\left(x_{1}\right) \oplus \psi\left(x_{2}\right)\end{cases}$

There is on $\operatorname{Hom}_{A}\left(M_{1}, M_{2}\right)$ an obvious tropical A-semimodule structure :

$(a \odot \psi)(x)=a \odot(\psi(x))$ and $\left(\psi_{1} \oplus \psi_{2}\right)(x)=\psi_{1}(x) \oplus \psi_{2}(x)$.

For all $\psi \in \operatorname{Hom}_{A}\left(M_{1}, M_{2}\right), \psi(0)=0$; let ker $\psi=\left\{x \in M_{1}: \psi(x)=0\right\}$, it is a sub-semimodule of $M_{1}$.

Elements of $\operatorname{Hom}_{A}\left(M_{1}, M_{2}\right)$ are convexity preserving maps (as previously defined) but also, the image $\psi(C)$ of an element $C \in \mathcal{C}_{\text {trop }}\left(M_{1}\right)$ by a map $\psi \in \operatorname{Hom}_{A}\left(M_{1}, M_{2}\right)$ belongs to $\mathcal{C}_{\text {trop }}\left(M_{2}\right)$.

A map $\theta: C_{1} \rightarrow M_{2}$ from $C_{1} \in \mathcal{C}_{\text {trop }}\left(M_{1}\right), C_{1} \neq \emptyset$, to $M_{2}$ is A-affine if, for all $x_{1}, x_{2} \in C_{1}$ and for all $a_{1}, a_{2} \in A$ such that $a_{1} \oplus a_{2}=1$

$$
\theta\left(\left(a_{1} \odot x_{1}\right) \oplus\left(a_{2} \odot x_{2}\right)\right)=a_{1} \theta\left(x_{1}\right) \oplus a_{2} \theta\left(x_{2}\right)
$$

The set $\operatorname{Aff}_{A}\left(C_{1}, M_{2}\right)$ of $A$-affine maps from $C_{1}$ to $M_{2}$ is, with respect to the pointwise $\odot$ and $\oplus$ operations, an $A$-semimodule (recall that the $\odot$ operation on $A$ is assumed to be commutative). The image of a convex subset of $C_{1}$ by $\theta \in \operatorname{Aff}_{A}\left(C_{1}, M_{2}\right)$ is convex and the inverse images of convex subset of $M_{2}$ is a convex subset of $C_{1}$.

(2) Let us say that a subset $K$ of an $A$-semimodule $M$ is a cone in $\mathbf{M}$ if:

$\begin{cases}(1) \forall\left(x_{1}, x_{2}\right) \in K \times K & x_{1} \oplus x_{2} \in K \\ (2) \forall(a, x) \in A_{\star} \times K & a \odot x \in K\end{cases}$

A cone in $M$ is a convex subset of $M$ (if $a_{1} \oplus a_{2}=1$ then either $a_{1} \neq 0$ or $a_{2} \neq 0$ ). If $K$ is a cone in $M$ then $K \cup\{0\}$ is a subsemimodule of $M$ and if $A$ is a semifield and $L$ is a sub-semimodule of $M$ then $L_{\star}=L \backslash\{0\}$ is a cone in $M$. For example, given a nonempty set $X$, let, for all $a \in A, \mathrm{c}_{a} \in A^{X}$ be the constant map $\mathrm{c}_{a}(x)=a$; $A^{X}$, with the pointwise operations is an $A$-semimodule and if $A$ is a semifield $\left\{\mathrm{c}_{a}: a \in A_{\star}\right\}$ is a cone in $A^{X}$ (which we identify with $A_{\star}$ ) and $A_{\star}^{X}$ is also a cone in $A^{X}$ which contains $A_{\star}$.

Given two cones $K_{1} \subset M_{1}$ and $K_{2} \subset M_{2}$ let $\operatorname{Hom}_{A}\left(K_{1}, K_{2}\right)$ be the set of maps $\psi: K_{1} \rightarrow K_{2}$ such that 


$$
\begin{cases}(1) \forall\left(x_{1}, x_{2}\right) \in K_{1} \times K_{1} & \psi\left(x_{1} \oplus x_{2}\right)=\psi\left(x_{1}\right) \oplus \psi\left(x_{2}\right) \\ (2) \forall(a, x) \in A_{\star} \times K_{1} & \psi(a \odot x)=a \odot \psi(x)\end{cases}
$$

If $A$ is a semifield then the set $\operatorname{Hom}_{A}\left(K_{1}, K_{2}\right)$ is itself a cone in the $A$-semimodule $A^{X}$.

If $K$ is a subcone of $A_{\star}^{X}$ such that c $c_{1} \in K$ let

$$
\mathcal{M}_{A}(K)=\left\{\psi \in \operatorname{Hom}_{A}\left(K, A_{\star}\right): \psi\left(\mathrm{c}_{1}\right)=1\right\}
$$

Clearly, if $\psi \in \mathcal{M}_{A}(K)$ then, for all $a \in A_{\star}, \psi\left(\mathrm{c}_{a}\right)=a$.

To each $x \in X$ one can associate the map "evaluation at $x$ ", $\psi \rightarrow \delta_{x}(\psi)=\psi(x)$; clearly, $\delta_{x} \in \operatorname{Hom}_{A}\left(K, A_{\star}\right)$ and, $\delta_{x}\left(\mathrm{c}_{1}\right)=1$; this shows that $\mathcal{M}_{A}(K)$ is not empty.

If $\psi_{1}$ and $\psi_{2}$ are in $\mathcal{M}_{A}(K)$ then $\psi_{1} \oplus \psi_{2}$ is in $\mathcal{M}_{A}(K)$ and, if $A$ is a semifield then, for all $a \in A_{\star}$ and all $\psi \in \mathcal{M}_{A}(K), a \odot \psi$ is in $\mathcal{M}_{A}(K)$.

In other words, if $A$ is a semifield then $\mathcal{M}_{A}(K)$ is a cone in the $A$-semimodule $A^{K}$, as a matter of facts a subcone of $A_{\star}^{K}$.

If one takes $A=\mathbb{R} \cup\{-\infty\}$ and if $X$ is a compact topological space then $\mathcal{C}(X)$, the space on real valued continuous functions on $X$, is a maxplus-cone in $(\mathbb{R} \cup\{-\infty\})^{X}$ then $\mathcal{M}_{A}(\mathcal{C}(X))$ is the cone of Maslov's measures on $X$ or idempotent measures on $X$, for its topological structure when $X$ is a compact metric space see [3]. (4) Let us denote by $\mathcal{C}_{\text {trop }}^{\bullet}(M)$ the set of nonempty convex sets of a tropical semimodule $M$ over a tropical (commutative) semiring $A$; define on $\mathcal{C}_{\text {trop }}^{\bullet}(M)$ the operations $\odot$ and $\oplus$ as follows: $\left\{\begin{array}{l}a \odot C=\{a \odot x: x \in C\} \\ C_{1} \oplus C_{2}=\left\{u \oplus v:(u, v) \in C_{1} \times C_{2}\right\}\end{array}\right.$

For $a \in A$ and $C_{1}, C_{2} \in \mathcal{C}_{\text {trop }}^{\bullet}(M),\left\{\begin{array}{l}C_{1} \oplus C_{2} \in \mathcal{C}_{\text {trop }}^{\bullet}(M) \text { and } \\ a \odot\left(C_{1} \oplus C_{2}\right)=a \odot C_{1} \oplus a \odot C_{2}\end{array}\right.$

and, for $a, b \in A$ and $C \in \mathcal{C}_{\text {trop }}^{\bullet}(M),\left\{\begin{array}{l}a \odot C \in \mathcal{C}_{\text {trop }}^{\bullet}(M) \\ (a \odot b) \odot C=a \odot(b \odot C) \\ 1 \odot C=C \text { and } 0 \odot C=\{0\}\end{array}\right.$

That $\left(C_{1} \oplus C_{2}\right) \oplus C_{3}=C_{1} \oplus\left(C_{2} \oplus C_{3}\right)$ is also clear as well as the inclusion $(a \oplus b) \odot C \subset(a \odot C) \oplus(b \odot C)$. If $A$ is a semifield then $(a \odot C) \oplus(b \odot C) \subset(a \oplus b) \odot C$.

Indeed if $z=a \odot x \oplus b \odot y$ with $x, y \in C$ either $a \oplus b=0$ or $a \oplus b \neq 0$. In the first case $a=b=0$ and $(a \oplus b) \odot C=0 \odot C=\{0\}$ while $(a \odot C) \oplus(b \odot C)=\{0\} \oplus\{0\}=\{0\}$. If $a \oplus b \neq 0$ let $c=(a \oplus b)^{\text {inv }}$; then $(c \odot a) \odot x \oplus(c \odot b) \odot y \in C$ and $z=(a \oplus b)((c \odot a) \odot x \oplus(c \odot b) \odot y) \in(a \oplus b) \odot C$.

In conclusion: if $A$ is a semifield then $\mathcal{C}_{\text {trop }}^{\bullet}(M)$, endowed with elementwise multiplication by elements of $A$ and elementwise addition is a tropical semimodule over $A$.

(5) Let $\mathcal{P}_{\text {oly }}^{\bullet} \cdot(M)$ be the set of nonempty tropical polytopes of the $A$-semimodule $M$; it is a subset of $\mathcal{C}_{\text {trop }}^{\bullet}(M)$ to which $\{0\}$ belongs. If $P=[S]$ where $S$ is finite nonempty subset of $M$ then, for all $a \in A$, $a \odot P=[\{a \odot x: x \in S\}]$ an therefore $a \odot P$ is a polytope. What might not be at first so clear is that $P_{1} \oplus P_{2}$ is a polytope if $P_{1}$ and $P_{2}$ are polytopes. We will show that this is the case if $A$ is a totally ordered semifield and therefore, if $A$ is a totally ordered semifield then $\mathcal{P}$ oly $\dot{\varphi}_{\text {trop }}(M)$ is a tropical semimodule over $A$ as a matter of fact, a tropical subsemimodule of $\mathcal{C}_{\text {trop }}^{\bullet}(M)$.

The set $T \subset M$ being finite and nonempty let us write $\bigoplus T$ for $\bigoplus_{x \in T} x$. Given two nonempty finite subsets $S_{1}$ and $S_{2}$ of $M$ let

$$
\left.\left[S_{1} \mid S_{2}\right]=\left[\oplus T_{1} \oplus \bigoplus T_{2}: T_{1} \times T_{2} \subset S_{1} \times S_{2} \quad T_{1} \times T_{2} \neq \emptyset\right\}\right]
$$

We have $\left[S_{1} \mid S_{2}\right] \in \mathcal{P}_{\text {oly }}^{\bullet} \bullet(M)$ and $\left.\left[S_{1} \mid S_{2}\right] \subset \llbracket S_{1}\right] \oplus\left[S_{2}\right]$. Let us show by induction on the cardinality of $S_{1}$ that $\left[S_{1}\right] \oplus \llbracket S_{2} \rrbracket \subset\left[S_{1} \mid S_{2}\right]$. Let $S_{1}=\left\{x_{1}, \cdots, x_{k}\right\}$ and $S_{2}=\left\{y_{1}, \cdots, y_{m}\right\}, u=\oplus_{i=1}^{k} a_{i} x_{i}, v=\oplus_{j=1}^{m} b_{j} y_{j}$ and $\oplus_{i=1}^{k} a_{i}=\oplus_{j=1}^{m} b_{j}=1$.

If $k=1$ then $u \oplus v=x_{1} \oplus\left(\oplus_{j=1}^{m} b_{j} \odot y_{j}\right)=\oplus_{j=1}^{m} b_{j} \odot\left(x_{1} \oplus y_{j}\right) \in\left[S_{1} \mid S_{2}\right]$.

Let $k=n+1$. By hypothesis $A$ is totally ordered and $\oplus_{i=1}^{n+1} a_{i}=1$, one of the $a_{i}$ is therefore equal to 1 ; without loss of generality one can assume that $a_{1}=1$. Let $u_{1}=\oplus_{i=1}^{n} a_{i} x_{i}$; from $\oplus_{i=1}^{n} a_{i}=1$ and the induction 
hypothesis we have $u_{1} \oplus v \in\left[S_{1} \backslash\left\{x_{n+1}\right\}\left|S_{2} \rrbracket \subset \llbracket S_{1}\right| S_{2}\right]$.

Let us write $u_{1} \oplus v=\oplus_{j=1}^{l} d_{j}\left(\bigoplus T_{1_{j}} \oplus \bigoplus T_{2_{j}}\right)$ where $\oplus_{j=1}^{l} d_{j}=1$ and, for all $j, T_{1_{j}}$ is a nonempty subset of $S_{1} \backslash\left\{x_{n+1}\right\}$ and $T_{2_{j}}$ is a nonempty subset of $S_{2}$.

Then $u_{1} \oplus v \oplus x_{n+1}=\oplus_{j=1}^{l} d_{j}\left(\oplus\left[T_{1_{j}} \oplus x_{n+1}\right] \oplus \oplus T_{2_{j}}\right)$ and therefore $u_{1} \oplus v \oplus x_{n+1} \in\left[S_{1} \mid S_{2}\right]$.

$>$ From $1 \oplus a_{m+1}=1$ we have $\left(u_{1} \oplus v\right) \oplus\left[a_{m+1} \odot\left(u_{1} \oplus v\right)\right]=u_{1} \oplus v$ and from the convexity of $\left[S_{1} \mid S_{2}\right]$ we have $\left(u_{1} \oplus v\right) \oplus a_{m+1}\left(u_{1} \oplus v \oplus x_{n+1}\right) \in\left[S_{1} \mid S_{2}\right]$. But $\left(u_{1} \oplus v\right) \oplus a_{m+1}\left(u_{1} \oplus v \oplus x_{n+1}\right)=\left(u_{1} \oplus v\right) \oplus a_{m+1}\left(u_{1} \oplus v\right) \oplus$ $a_{m+1} x_{m+1}=\left(u_{1} \oplus v\right) \oplus a_{m+1} x_{m+1}=u \oplus v$.

For the topological structure of the space of nonempty compact maxplus convex subsets of a power of the real line see [4] and the references cited therein.

\section{Convexity And TOpology}

Given a set $X$ a convexity $\mathcal{C}$ and a topology $\tau$ what kind of meaningful relationship can one impose between $\mathcal{C}$ and $\tau$ ? Connectedness of polytopes seems natural but, in itself, might not be sufficient to yield the fixed point property or other properties related to convexity in normed or locally convex topological vector spaces. Nonetheless, we will recall at the end of this section that connectedness is still good enough to capture some of the fundamental results of classical nonlinear analysis.

The standard $n$ dimensional simplex, that is $\left\{\left(t_{0}, \cdots, t_{n}\right) \in[0,1]^{n+1}: \sum_{i=0}^{n} t_{i}=1\right\}$ will be denoted, as usual, by $\Delta_{n}$ : the boundary $\partial \Delta_{n}$ of $\Delta_{n}$ is $\left\{\left(t_{0}, \cdots, t_{n}\right) \in \Delta_{n}: \prod_{i=0}^{n} t_{i}=0\right\}$. The family of nonempty subsets of $\{0, \cdots, n\}$ is denoted by $\langle n\rangle$ and, for $J \in\langle n\rangle, \Delta_{n, J}=\left\{\left(t_{0}, \cdots, t_{n}\right) \in \Delta_{n}: \forall i \notin J t_{i}=0\right\}$ and $\Delta_{n}^{-j}=\left\{\left(t_{0}, \cdots, t_{n}\right) \in \Delta_{n}: t_{j}=0\right\}$.

Let us recall that a topological space $Z$ is homothopically trivial if, for all $n \in \mathbb{N}$ and all continuous map $g: \partial \Delta_{n} \rightarrow Z$ there exists a continuous map $G: \Delta_{n} \rightarrow Z$ whose restriction to $\partial \Delta_{n}$ is $g$. A topological space $Z$ is contractible if there exists a continuous map $h:[0,1] \times Z \rightarrow Z$ such that $h(0,-): Z \rightarrow Z$ is constant and $h(1,-): Z \rightarrow Z$ is the identity map; contractible spaces are homothopically trivial.

A convex topological space is a triple $(X, \tau, \mathcal{C})$ where $\tau$ is a topology on $X$ and $\mathcal{C}$ is a convexity on $X$. Two convex topological spaces $\left(X_{1}, \tau_{1}, \mathcal{C}_{1}\right)$ and $\left(X_{2}, \tau_{2}, \mathcal{C}_{2}\right)$ are homeomorphic convex topological spaces if there exists an homeomorphism $\varphi:\left(X_{1}, \tau_{1}\right) \rightarrow\left(X_{2}, \tau_{2}\right)$ which is also an isomorphism of the convex spaces $\left(X_{1}, \mathcal{C}_{1}\right)$ and $\left(X_{2}, \mathcal{C}_{2}\right)$. For example, $x \mapsto \ln (x)$ is an homeomorphism from the $\mathbb{B}$-convex space $\mathbb{R}_{++}^{n}$ to the maxplus convex space $\mathbb{R}^{n}$; one could extend this homeomorphism from $\mathbb{R}_{+}^{n}$ to $(\mathbb{R} \cup\{-\infty\})^{n}$ by taking as a neighbourhood base for $-\infty$ in $\mathbb{R} \cup\{-\infty\}$ the family of complements of closed half rays $[r,+\infty[, r \in \mathbb{R}$; which makes $(\mathbb{R} \cup\{-\infty\})^{n}$ homeomorphic to $\mathbb{R}_{+}$.

It is not hard to see that $\mathbb{B}$-polytopes in $\mathbb{R}_{+}^{n}$ are compact and contractible, see [10], and obviously metrizable ; the same is therefore true of maxplus-polytopes in $\mathbb{R}^{n}$.

A family of topological results has played a fundamental role in nonlinear analysis and mathematical economics; these results pertain mainly to the existence of a fixed point (for a single valued or a multivalued map), to the existence of a continuous selection (or an approximate continuous selection) for a multivalued map or to the nonvacuity of the intersection of a given family of sets. Most of these results can be extended to topological convex spaces whose polytopes are homothopically trivial. One of these results is as easily stated as it is fundamental, in its classical version it can be seen as an avatar of Brouwer's fixed point theorem: it is the Knaster-Kuratowski-Mazurkiewicz theorem, also known as the KKM Lemma, whose standard form can be found in [15], [1], [2], [7] or [28] along with its various forms and applications. The classical "KKM Lemma" reads as follows:

( $\mathbf{K K M ) ~ I f ~}\left\{F_{0}, \cdots, F_{n}\right\}$ is a family of closed sets of $\Delta_{n}$ such that, for all $J \in\langle n\rangle, \Delta_{n J} \subset \cup_{j \in J} F_{j}$ then $\cap_{j=0}^{n} F_{j} \neq \emptyset$. 
Lassonde noticed that the KKM Lemma holds if, instead of being closed, all the $F_{i}$ are open [25]; but see also [16] and [27]. We will still refer to the KKM Lemma for either a closed or an open covering as the "classical KKM".

\subsection{Some basic intersection theorems in topological convex spaces}

Given a topological convex space $(X, \tau, \mathcal{C})$ let us say that a subset $A$ of $X$ is finitely closed (resp. open) if, for all polytope $P \in \mathcal{C}, A \cap P$ is closed (resp. open) in $P$ with respect to the induced topology.

Proposition 4.1.1 (KKM). In a topological convex space $(X, \tau, \mathcal{C})$ with homothopically trivial polytopes let $\left\{F_{0}, \cdots, F_{n}\right\}$ be a family of subsets of $X$ all of which are finitely closed or all of which are finitely open. Then $\cap_{i=0}^{n} F_{i} \neq \emptyset$ if and only if there exists a family of points $\left\{x_{0}, \cdots, x_{n}\right\}$ of $X$, not necessarily distincts, such that, for all nonempty subset $J \subset\{0, \cdots, n\},\left[\left\{x_{j}: j \in J\right\}\right] \subset \cup_{j \in J} F_{j}$.

Proof. Let $P=\left[\left\{x_{0}, \cdots, x_{n}\right\} \rrbracket\right.$ and, for all $J \in\langle n\rangle$, let $P_{J}=\left[\left\{x_{i}: i \in J\right\} \rrbracket\right.$.

As in [18], Theorem 1, one shows that there exists a continuous map $\theta: \Delta_{n} \rightarrow P$ such that, $\forall J \in\langle n\rangle, \theta\left(\Delta_{n, J}\right) \subset\left[\left\{x_{i}: i \in J\right\}\right]$.

Let $M_{j}=\theta^{-1}\left(P \cap F_{j}\right)$; from $P \subset \cup_{j=0}^{n} F_{j}$ we have $\Delta_{n}=\cup_{j=0}^{n} M_{j}$ and also $\Delta_{n, J} \subset \cup_{j \in J} M_{j}$ and finally, from the classical KKM Lemma, $\cap_{j=0}^{n} M_{j} \neq \emptyset$.

Proposition 4.1.2 (Alexandrov-Pasynkov). In a topological convex space $(X, \tau, \mathcal{C})$ with homothopically trivial polytopes let $\left\{F_{0}, \cdots, F_{n}\right\}$ be a family of subsets of $X$ all of which are finitely closed or all of which are finitely open. If there exists a family of points $\left\{x_{0}, \cdots, x_{n}\right\}$ of $X$, not necessarily distincts, such that, $\left[\left\{x_{0}, \cdots, x_{n}\right\} \rrbracket \subset\right.$ $\cup_{j=0}^{n} F_{j}$ and, for all $j \in\{0, \cdots, n\},\left[\left\{x_{i}: i \neq j\right\}\right] \subset F_{j}$ then $\cap_{i=0}^{n} F_{i} \neq \emptyset$.

Proof. Let $P^{-j}=\left[\left\{x_{i}: i \neq j\right\}\right]$; all the other symbols retain the meaning they had in the previous proposition. Since $P \subset \cup_{j=0}^{n} F_{j}$ we have $\Delta_{n}=\cup_{j=0}^{n} M_{j}$ and we also have, for all $j \in\{0, \cdots, n\}, \Delta_{n}^{-j} \subset M_{j}$ and finally, from the classical Alexandrov-Pasynkov Theorem, $\cap_{j=0}^{n} M_{j} \neq \emptyset$.

Proposition 4.1.3 (Klee's Theorem). In a topological convex space $(X, \tau, \mathcal{C})$ with homothopically trivial polytopes let $\left\{C_{0}, \cdots, C_{n}\right\}$ be a family of convex sets of $X$ of which are finitely closed or all of which are finitely open. If, $\cup_{i=0}^{n} C_{i}$ is convex and, for all $j \in\{0, \cdots, n\}, \cap_{i \neq j} C_{i} \neq \emptyset$, then $\cap_{i=0}^{n} C_{i} \neq \emptyset$.

Proof. For each $i \in\{0, \cdots, n\}$ pick a point $x_{i} \in \cap_{i \neq j} C_{j}$; we then have

$\left[\left\{x_{0}, \cdots, x_{n}\right\}\right] \subset \cup_{j=0}^{n} C_{j}$ and, for all $j \in\{0, \cdots, n\},\left\{x_{i}: i \neq j\right\} \subset C_{j}$. From the Alexandrov-Pasynkov Theorem, $\cap_{i=0}^{n} M_{i} \neq \emptyset$ and consequently, $\cap_{i=0}^{n} C_{i} \neq \emptyset$.

Klee's Theorem [24] for the usual linear convexity was proved independently by Berge in 1959, [5] or [6], using the Hahn-Banach Theorem.

Let us say that a subset $S$ of a convex topological space $(X, \mathcal{C})$ is starshaped if there exists a point $x_{0} \in S$ such that, for all $x \in S, \llbracket x_{0}, x \rrbracket \subset S$; in which case we say that $S$ is starshaped at $x_{0}$. A convex set is starshaped. The next result is a topological version of Breen's Theorem [11].

Proposition 4.1.4 (Breen's Theorem). Let $(X, \tau, \mathcal{C})$ be a convex topological space in which starshaped subsets of polytopes are homothopically trivial. Let $\left\{U_{0}, \cdots, U_{n}\right\}$ be a family of subsets of $X$ all of which are finitely closed or all of which are finitely open. If, for all nonempty subsets $J \subset\{0, \cdots, n\}$ the set $\cup_{j \in J} U_{j}$ is starshaped then $\cap_{j=0}^{n} U_{j} \neq \emptyset$.

Proof. For each $J \in\langle n\rangle$ let $F_{J}=\cup_{j \in J} U_{j}$; choose $x_{J} \in F_{J}$ such that $F_{J}$ is starshaped at $x_{J}$. Let $P=\left[\left\{x_{J}\right.\right.$ : $J \in\langle n\rangle\}]$ and $S_{J}=P \cap F_{J}$; notice that $S_{J}$ is starshaped at $x_{J}$. Given $J_{1}, \cdots, J_{l} \in\langle n\rangle$ let $J=J_{1} \cup \cdots \cup J_{l}$ and notice that $S_{J_{1}} \cup \cdots \cup S_{J_{1}}=S_{J}$. The family $\left\{S_{J}: J \in\langle n\rangle\right\}$ is made of starshaped subsets of the polytopes $P$ and either all of its members are closed in $P$ or all of its members are open in $P$; furthermore, by hypothesis, 
all of the sets $S_{J}$ are homothopically trivial subsets of $P$. Enumerate $\langle n\rangle$ arbitrarily, $\langle n\rangle=\left\{J_{0}, \cdots, J_{m}\right\}$; since, for all $\left\{J_{i_{1}}, \cdots, J_{i_{k}}\right\}, \cup_{j=1}^{k} S_{J_{i_{j}}}=S_{J_{l}}$ with $J_{l}=\cup_{j=1}^{k} J_{i_{j}}$ we see that $\cup_{j=1}^{k} S_{J_{i_{j}}}$ is a starshaped subset of $P$.

For all $\lambda \in\{0, \cdots, m\}$, let $T_{\lambda}=S_{J_{\lambda}}=\cup_{j \in J_{\lambda}} S_{j} ; T_{\lambda}$ is an homothopically trivial subset of $P$ and the family $\left\{T_{\lambda}: \lambda \in\{0, \cdots, m\}\right\}$ is union closed.

For all $\Lambda \in\langle m\rangle$ let $G_{\Lambda}=\cup_{\lambda \in \Lambda} T_{\lambda}$. If $\Lambda \subset \Lambda^{\prime}$ then $G_{\Lambda} \subset G_{\Lambda^{\prime}}$ and these sets are homothopically trivial subsets of $P$. There exists a continuous map $\theta: \Delta_{m} \rightarrow P$ such that, for all $\Lambda \in\langle m\rangle, \theta\left(\Delta_{m, \Lambda}\right) \subset G_{\Lambda}$. Let $M_{\lambda}=\theta^{-1}\left(T_{\lambda}\right)$; notice that $\Delta_{m, \Lambda} \subset \cup_{\lambda \in \Lambda} M_{\lambda}$ and that, either all the sets $M_{\lambda}$ are closed in $\Delta_{m}$ or all of them are open in $\Delta_{m}$. By the standard KKM Theorem, $\cap_{\lambda=0}^{m} M_{\lambda} \neq \emptyset$ and therefore $\cap_{\lambda=0}^{m} T_{\lambda} \neq \emptyset$ that is $\cap_{J \in\langle n\rangle} S_{J} \neq \emptyset$ and a fortiori, $\cap_{J \in\langle n\rangle} F_{J} \neq \emptyset$ from which $\cap_{i=0}^{n} U_{i} \neq \emptyset$.

Breen's original result is on the one hand more precise than Proposition 4.1.4 and on the other hand less general. She considered a family $\mathcal{F}$ of nonempty compact starshaped sets in $\mathbb{R}^{d}$ and proved that $\cap \mathcal{F} \neq \emptyset$ if the union of every subfamily of $\mathcal{F}$ of $d+1$ or fewer members of $\mathcal{F}$ has starshaped union. On the other hand, Proposition 4.1.4 holds in arbitrary topological vector spaces. For a topological proof of Breen's original theorem see [22].

One can show that tropical starshaped sets in $\mathbb{R}^{d}$, that is maxplus starshaped sets, are homothopically trivial, as well as $\mathbb{B}$-convex (maxtimes) starshaped sets in $\mathbb{R}_{+}^{d}$. We leave aside for now the question of a completely analoguous "tropical Breen's Theorem" in $\mathbb{R}^{d}$ or in $\mathbb{R}_{+}^{d}$.

There is another Proposition whose proof is somewhat similar to that of Proposition 4.1.4; to keep this section at a reasonable length the proof will not be given here.

Proposition 4.1.5. Let $(X, \tau, \mathcal{C})$ be a convex topological space in which starshaped subsets of polytopes are homothopically trivial. Let $\left\{U_{0}, \cdots, U_{n}\right\}$ be a family of subsets of $X$ all of which are finitely closed or all of which are finitely open. If, for all nonempty subsets $J \subset\{0, \cdots, n\}$ of cardinality at most $n$ the set $\cap_{j \in J} U_{j}$ is starshaped and if $\cup_{j=0}^{n} U_{j}$ is starshaped then $\cap_{j=0}^{n} U_{j} \neq \emptyset$.

\subsection{Fixed points theorems}

The results of this section, given without proofs, rely on the fact that maxplus polytopes in $\mathbb{R}^{n}$ and $\mathbb{B}$ polytopes in $\mathbb{R}_{+}^{n}$ are contractible, and therefore homothopically trivial.

The first result is a tropical version of the Fan-Browder Theorem, page 143 in [15].

Proposition 4.2.1. Let $C$ be either a compact maxplus convex set in $\mathbb{R}^{n}$ or a compact $\mathbb{B}$-convex set in $\mathbb{R}_{+}^{n}$ and let $T: C \rightarrow C$ be a multivalued map with closed nonempty maxplus (respectively, $\mathbb{B}$ ) convex values. If, for all $y \in C$, the set $T^{-1} y=\{x \in C: y \in T x\}$ is open in $C$ then there exists $\bar{x} \in C$ such that $\bar{x} \in T \bar{x}$.

Proof. It is a consequence of Proposition 4.1.1; see [19] Theorem 3 for the details.

An arbitrary product $\prod_{i=1}^{n}\left[a_{i}, b_{i}\right]$ of real intervals is a $\mathbb{B}$-convex set in $\mathbb{R}_{+}^{n}$ consequently, an arbitrary nonempty $\mathbb{B}$-convex set in $\mathbb{R}_{+}^{n}$ has a neighbourhood base consisting of $\mathbb{B}$-convex sets ; $\mathbb{B}$-convex set are obviously metrizable. By the corollary on page 259 of [19], arbitrary nonempty $\mathbb{B}$-convex set in $\mathbb{R}_{+}^{n}$ are absolute retracts. The same is true of arbitrary nonempty maxplus convex sets in $\mathbb{R}^{n}$. Since we are here in finite dimention we could also reach the same conclusion from the fact that $\mathbb{B}$-convex sets are contractible and locally contractible.

Proposition 4.2.2. Let $C$ be either a maxplus convex set in $\mathbb{R}^{n}$ or a $\mathbb{B}$-convex set in $\mathbb{R}_{+}^{n}$ and $f: C \rightarrow C$ a continuous map whose image is contained in a compact subset of $C$. Then $f$ has a fixed point.

Proof. From the Generalized Schauder Theorem, page 165 in [15].

We close this section with Kakutani's Fixed Theorem in maxplus or $\mathbb{B}$-convexity. First, in $\mathbb{R}_{+}^{n}$ open balls with respect to the $\|\cdot\|_{\infty}$ norm are $\mathbb{B}$-convex sets and, for all $\mathbb{B}$-convex subset $C$ of $\mathbb{R}_{+}^{n}$ and all $r>0$ the 
$r$-neighbourhood of $C$ with respect to $\|\cdot\|_{\infty}$ are $\mathbb{B}$-convex; in the language of $[19]$, $\mathbb{B}$-convex sets are l.c. spaces. The same is true of maxplus convex sets in $\mathbb{R}^{n}$.

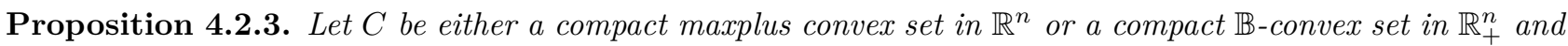
$T: C \rightarrow C$ an an upper semicontinuous multivalued map with nonempty closed maxplus (respectively, $\mathbb{B})$ convex values. Then, there exists $\bar{x} \in C$ such that $\bar{x} \in T \bar{x}$.

Proof. Page 262 of [19].

\subsection{Back to the begining}

The two previous sections lead naturally to the following question: when does a convex topological space have homothopically trivial polytopes? As we have already observed, maxplus polytopes in $\mathbb{R}^{n}$, or $\mathbb{B}_{-p o l y t o p e s}$ in $\mathbb{R}_{+}^{n}$ are homothopically trivial, as well as usual linear polytopes, and all of this can easily be shown by hand without appealing to any general theory. Let us nonetheless reformulate our question as follows: what kind of "compatibility" properties should link the convexity and the topology of a given convex topological space $(X, \tau, \mathcal{C})$ in order to have homothopically trivial polytopes ? Of course, we would like the standard tropical convexities, maxplus in $\mathbb{R}^{n}$ or maxtimes in $\mathbb{R}_{+}^{n}$, to have those properties. Two answers will be given, one geometric, in terms of the existence of a compatible geodesic structure, and one topological. For simplicity, we will restrict our attention to metric spaces eventhough it is not necessary, and even not recommanded, think of convex sets in locally convex topological vector spaces. In everything that follows $(X, d)$ is a metric space and $\mathcal{C}$ is a convexity on $X$.

\subsubsection{Uniform geodesic convexities}

Let us say that $(X, d, \mathcal{C})$ is a uniform geodesic space if $\mathcal{C}$ is an interval convexity and if there exists a continu-

ous function $\lambda: X \times X \times[0,1] \rightarrow X$ such that $\forall\left(x_{0}, x_{1}, t, s\right) \begin{cases}(1) & \lambda\left(x_{0}, x_{1}, 0\right)=x_{0} \text { and } \lambda\left(x_{0}, x_{1}, 1\right)=x_{1} \\ (2) & d\left(\lambda\left(x_{0}, x_{1}, t\right), \lambda\left(x_{0}, x_{1}, s\right)\right)=|t-s| d\left(x_{0}, x_{1}\right) \\ (3) & \lambda\left(x_{0}, x_{1},[0,1]\right)=\left[x_{0}, x_{1}\right]\end{cases}$

If $(X, d, \mathcal{C})$ is a uniform geodesic space then all nonempty convex subsets of $X$ are, with respect to the induced convexity and the induced metric, uniform geodesic spaces. These are indeed very strong conditions, and stronger than they need to be, but as they stand they hold for maxplus convexity in $\mathbb{R}^{n}$ and $\mathbb{B}$-convexity in $\mathbb{R}_{++}^{n}$. In the first case, the metric is given $d(x, y)=\|x-x \vee y\|_{\infty}+\|y-x \vee y\|_{\infty}$, one can check that it is the so called Hilbert affine distance (Stephane Gaubert showed to the author that maxplus segments in $\mathbb{R}^{n}$ are geodesics for the Hilbert affine distance) that is, for $x, y \in \mathbb{R}^{n}, d(x, y)=\max _{i}\left\{0, x_{i}-y_{i}\right\}+\max _{i}\left\{0, y_{i}-x_{i}\right\}$.

Conditions (1) and (3) imply together that starshaped sets are homothopically trivial, as a matter of fact they are contractible.

In a uniform geodesic space one can naturally define the midpoint function

$\mu: X \times X \rightarrow X$ by $\mu\left(x_{0}, x_{1}\right)=\lambda\left(x_{0}, x_{1}, 1 / 2\right)$. From $(2)$ we have $d\left(x_{0}, \mu\left(x_{0}, x_{1}\right)\right)=d\left(x_{1}, \mu\left(x_{0}, x_{1}\right)\right)=$ $1 / 2 d\left(x_{0}, x_{1}\right)$.

Proposition 4.3.1. In a uniform geodesic space $(X, d, \mathcal{C})$ a closed set $C \subset X$ is convex if and only if it contains the midpoint of any two of its points, that is $\mu(C \times C) \subset C$.

Proof. The nontrivial part of the proof is done by induction on the set of dyadic numbers in the real interval $[0,1]$.

One can see without too much difficulty that, for all maxplus convex segments $[x, y] \subset \mathbb{R}^{n},[x, y]=[x, x \oplus y] \cup$

$[x \oplus y, y]$ and therefore, a subset $C$ of $\mathbb{R}^{n}$ is maxplus convex if and only if $\left\{\begin{array}{l}\left.(1) \quad \forall x, y \in C x \oplus y \in C \text { (C is a subsemilattice of } \mathbb{R}^{n}\right) \\ (2) \quad \forall x, y \in C x \leq y \Rightarrow[x, y] \subset C .\end{array}\right.$ 
From this remark and Proposition 4.3.1 one gets the following caracterization of closed maxplus convex subsets of $\mathbb{R}^{n}$.

Proposition 4.3.2. A closed subset $C$ of $\mathbb{R}^{n}$ is maxplus convex if and only if $\left\{\begin{array}{l}(1) \quad C \text { is a subsemilattice of } \mathbb{R}^{n} \\ (2) \quad \forall x, y \in C x \leq y \Rightarrow \mu_{\text {max }}(x, y) \in C \text {. }\end{array}\right.$ where $(x, y) \mapsto \mu_{\max }(x, y)$ is the midpoint function on $\mathbb{R}^{n}$ associated to the Hilbert affine metric.

One can show that, if $x \leqslant y$ then $\mu_{\max }(x, y)_{i}=\max \left\{x_{i}, y_{i}-\frac{\max _{1 \leq j \leq n}\left\{y_{j}-x_{j}\right\}}{2}\right\}$.

\subsubsection{Uniform convexities}

We restrict the discussion to metric spaces, but uniform topological spaces would do as well. Let $\mathcal{C}$ be a convexity on a metric space $(X, d)$ such that polytopes are compact. The convexity is completely specified by the convex hull operator restricted to the set of nonempty finite subsets of $X$; let $\langle X\rangle$ be that space and let $\mathcal{K}(X)$ be the space of nonempty compact subsets of $X$. Both $\langle X\rangle$ and $\mathcal{K}(X)$ are metric spaces with respect to the Hausdorff metric associated to the metric $d$ on $X$. Let us say that $(X, d, \mathcal{C})$ is a uniform convex space if the convex hull operator $\left\{x_{0}, \cdots, x_{n}\right\} \mapsto\left[x_{0}, \cdots, x_{n}\right]$ is uniformly continuous from $\langle X\rangle$ to $\mathcal{K}(X)$. One can show that $\mathbb{B}$-convexity on $\mathbb{R}_{+}^{n}$ is a uniform convexity; the same is true of maxplus convexity on $\mathbb{R}^{n}$.

Proposition 4.3.3. Let $(X, d, \mathcal{C})$ be a convex space with compact polytopes. If $(X, d, \mathcal{C})$ is a uniform convex space, if polytopes are connected and if the Kakutani Separation Property holds then convex sets are homothopically trivial.

The proof is somewhat too involved to be presented here; it can be found in [20], for uniform topological spaces, or in [30] for metric spaces. All of the classical selection, extension and fixed point theorems for locally convex and normed spaces, as well as some topological properties, like being an absolute retract, hold in the context of uniform convexities. It has been said at the begining of this discussion that a convexity has the Kakutani Separation Property if and only if arbitrary polytopes have it; therefore, all the results alluded to follow from properties of polytopes ("finite dimensional properties") and one global property: uniform continuity of the convex hull operator.

\section{REFERENCES}

[1] J.P. Aubin, Optima and Equilibria, An Introduction to Nonlinear Analaysis, second edition, Graduate Texts in Mathematics 140, Springer, 1998.

[2] J.P. Aubin, L'analyse non linéaire et ses motivations économiques, Masson, 1984.

[3] L. Bazylevych, D. Repovs, M. Zarichnyi, Spaces of idempotent measures of compact metric spaces, Top. and its App. 157 (2010) 136-144.

[4] L. Bazylevych, D. Repovs, M. Zarichnyi, Hyperspaces of max-plus convex subsets of power of the real line, J. Math. Anal. App. 394, 2 (2012), 481-487.

[5] C. Berge, Espaces topologiques et fonctions multivoques, Dunod, 1962.

[6] C. Berge, Topological spaces, Dover publications, 1997.

[7] K. Border, Fixed points theorems with applications to economics and game theory, Cambridge Univ. Press, 1985.

[8] W. Briec, C. Horvath And A. Rubinov, Separation in $\mathbb{B}$-convexity, Pacific Journal of Optimization, Vol.1, $\mathrm{n}^{\circ} 1$ (2005), 13-30.

[9] W. Briec, C. Horvath, Halfspaces and Hahn-Banach like properties in $\mathbb{B}$-convexity and max-plus convexity, Pacific Journal of Optimization, Vol.4, $\mathrm{n}^{\circ} 2$ (2008), 293-317.

[10] W. Briec, C. Horvath, Nash points, Ky Fan inequality and equilibria of abstract economies in Max-Plus and $\mathbb{B}$-convexity, J. Math. Anal. App. 341 (2008) 188-199.

[11] M. BreEn, Starshaped unions and nonempty intersections of convex sets in $\mathbb{R}^{d}$, Proc. Amer. Math. Soc., Vol.108, $\mathrm{n}^{\circ} 4(1990)$, $817-820$.

[12] G. Cohen, S. Gaubert And J.P. Quadrat, Hahn-Banach separation theorem for max- plus semimodules, in E. Rofman J.L. Menaldi and A. Sulem, editors, Optimal Control and Partial Differential Equations. IOS Press, 2001.

[13] G. Cohen, S. Gaubert, And J. P. Quadrat, Duality and separation theorems in idem- potent semimodules, Linear Algebra and Appl., 379 (2004) 395-422. 
[14] W.A. Coppel, Foundations of Convex Geometry, Cambridge University Press, 1998.

[15] J. Dugundu and A. Granas, Fixed Point Theory, Springer Verlag, 2003.

[16] K. FAn, Some properties of convex sets related to fixed point theorems, Math. Anal. 266 (1984), 519-537.

[17] Holmes, Geometric functional analysis and its applications, Springer Verlag 1975.

[18] C. Horvath, Contractibility and Generalized Convexity, JMAA, Vol. 156 (1991), 341-357.

[19] C. Honvath, Extension and selection theorems in topological spaces with a generalized convexity structure, Ann. Fac. Sci. Toulouse, Vol.II, nº 7 (1993) 253 - 269.

[20] C.Horvath, Toplogical convexities, selections and fixed points, Topology and its Applications, 155 (2008) 830-850.

[21] C. Horvath, A note on metric spaces with continuous midpoints, Annals of the Academy of Romanian Scientists, vol. $1, \mathrm{n}^{\circ}$ 2 (2009), 252-288.

[22] C. Horvath and M. Lassonde, Intersection of sets with n-connected unions, Proc. Amer. Math. Soc., Vol.125, $\mathrm{n}^{\circ} 4$ (1998), 1209-1214.

[23] K. Keimel, A. WieczoreK, Kakutani property of polytopes implies Kakutani property of the whole space, Math. Anal. App. $130(1988) 97-109$.

[24] V.L. KLEE, On certain intersections of convex sets, Canad. J. Math. 3 (1951), 272-275.

[25] M. Lassonde, Sur le principe KKM, Acad. Sci. Paris Série. I Math. 310 (1990) 573-576.

[26] V. Nitica And I. Singer, The structure of max-plus hyperplanes, Lin. Alg. and its App. 426 (2007) $382-414$

[27] M. H. Shiн, Covering properties of convex sets, Bull. London Math. Soc. 18 (1986) 57-59.

[28] S. Singh, B. Watson and P. SRivastava, Fixed point theory and best approximations: the KKM-map principle, Kluwer Academic Publishers, 1997.

[29] M. VAN DE Vel, Theory of convex structures, North Holland Mathematical Library, Vol. 50, 1993.

[30] M. VAn DE Vel, A selection theorem for topological convex structures, Trans. Amer. Math. Soc. 336 (2) (1993) $463-495$. 